\title{
Comparison of Clinical
}

Characteristics and Short-Term Prognoses Within Hospitalized Chronic Obstructive Pulmonary Disease Patients Comorbid With Asthma, Bronchiectasis, and Their Overlaps: Findings From the ACURE Registry

OPEN ACCESS

Edited by:

Chin Kook Rhee,

The Catholic University of Korea,

South Korea

Reviewed by:

Josuel Ora,

Policlinico Tor Vergata, Italy

Masaki Fujita,

Fukuoka University, Japan

*Correspondence:

Ting Yang

zryyyangting@163.com

Chen Wang

wangchen@pumc.edu.cn

Specialty section:

This article was submitted to

Pulmonary Medicine,

a section of the journal

Frontiers in Medicine

Received: 17 November 2021

Accepted: 26 January 2022

Published: 25 February 2022

Citation:

Lei J, Yang T, Liang C, Huang K, Wu S and Wang $C$ (2022) Comparison of

Clinical Characteristics and

Short-Term Prognoses Within

Hospitalized Chronic Obstructive

Pulmonary Disease Patients

Comorbid With Asthma,

Bronchiectasis, and Their Overlaps: Findings From the ACURE Registry.

Front. Med. 9:817048.

doi: 10.3389/fmed.2022.817048
Jieping Lei ${ }^{1,2,3,4,5}$, Ting Yang ${ }^{2,3,4,5,6 *}$, Chen Liang ${ }^{6}$, Ke Huang ${ }^{2,3,4,5,6}$, Sinan $W^{1,2,3,4,5}$ and Chen Wang 2,3,4,5,6,7,8* on behalf of the China Acute Exacerbation of Chronic Obstructive Pulmonary Disease Inpatient Registry (ACURE) Investigators

\begin{abstract}
${ }^{1}$ Data and Project Management Unit, Institute of Clinical Medical Sciences, China-Japan Friendship Hospital, Beijing, China, ${ }^{2}$ Department of Pulmonary and Critical Care Medicine, Center of Respiratory Medicine, China-Japan Friendship Hospital, Beijing, China, ${ }^{3}$ Institute of Respiratory Medicine, Chinese Academy of Medical Sciences, Beijing, China, ${ }^{4}$ National Clinical Research Center for Respiratory Disease, Beijing, China, ${ }^{5}$ National Center for Respiratory Medicine, Beijing, China, ${ }^{6}$ Chinese Alliance for Respiratory Diseases in Primary Care, Beijing, China, ${ }^{7}$ Chinese Academy of Medical Sciences, Peking Union Medical College, Beijing, China, ${ }^{8}$ Department of Respiratory Medicine, Capital Medical University, Beijing, China
\end{abstract}

Introduction: Real-world evidence and comparison among commonly seen chronic obstructive pulmonary disease (COPD) phenotypes, i.e., asthma-COPD overlap (ACO), bronchiectasis-COPD overlap (BCO), and their coexistence ( $\mathrm{ABCO}$ ) have not been fully depicted, especially in Chinese patients.

Methods: Data were retrieved from an ongoing nationwide registry in hospitalized patients due to acute exacerbation of COPD in China (ACURE).

Results: Of the eligible 4,813 patients with COPD, 338 (7.02\%), 492 (10.22\%), and 63 (1.31\%) were identified as ACO, BCO, and ABCO phenotypes, respectively. Relatively, the $\mathrm{ABCO}$ phenotype had a younger age with a median of 62.99 years [interquartile range (IQR): 55.93-69.48] and the COPD phenotype had an older age with a median of 70.15 years (IQR: $64.37-76.82)$. The BCO and COPD phenotypes were similar in body mass index with a median of $21.79 \mathrm{~kg} / \mathrm{m}^{2}$ (IQR: $\left.19.47-23.97\right)$ and $21.79 \mathrm{~kg} / \mathrm{m}^{2}$ (IQR: 19.49-24.22), respectively. The COPD phenotype had more male gender (79.90\%) and smokers $(71.12 \%)$ with a longer history of smoking (median: 32.45 years, IQR: 0.00-43.91). The ACO and ABCO phenotypes suffered more prior allergic episodes with a proportion of 18.05 and $19.05 \%$, respectively. The ACO phenotype exhibited a higher level of eosinophil and better lung reversibility. Moreover, the four phenotypes showed no significant difference neither in all-cause mortality, intensive care unit admission, length of hospital stay, and COPD Assessment Test score change during the index hospitalization, 
and nor in the day 30 outcomes, i.e., all-cause mortality, recurrence of exacerbation, all-cause, and exacerbation-related readmission.

Conclusions: The ACO, BCO, ABCO, and COPD phenotypes exhibited distinct clinical features but had no varied short-term prognoses. Further validation in a larger sample is warranted.

Keywords: chronic obstructive pulmonary disease, exacerbation, asthma, bronchiectasis, phenotype, heterogeneity, prognosis

\section{INTRODUCTION}

Patients with chronic obstructive pulmonary disease (COPD), comorbid with asthma (asthma-COPD overlap, ACO) or bronchiectasis (bronchiectasis-COPD overlap, BCO) as well as their coexistence (ABCO), are commonly seen phenotypes, which have been broadly discussed whether they were distinct disease entities, but there is no concluded consensus yet (1). The concept of "ACO" or "asthma + COPD" appeals as a great interest to investigators (2-11), and similar discussion applies to the research of bronchiectasis and COPD overlap (12-16). Few data was reported on the $\mathrm{ABCO}$ phenotype.

The prevalence of the above-mentioned phenotypes of COPD varied across studies. Alshabanat A et al. reported a pooled prevalence of ACO phenotype among patients with COPD was $27 \%$ and $28 \%$ in population- and hospital-based studies, respectively. Hosseini et al. (17) found that a pooled prevalence of ACO phenotype was $29.6 \%$ in patients with COPD. Zhou et al. (18) stated an ACO prevalence of $11.51 \%$ in Chinese patients with COPD. Ding et al. (19) reported an ACO prevalence of $18.6 \%$ in urban Chinese patients with COPD. Uchida et al. (20) summarized the prevalence of ACO phenotype that varied from 0.9 to $11.1 \%$ in the general population, from 11.1 to $61.0 \%$ in patients with asthma, and from 4.2 to $66.0 \%$ in patients with COPD (21). For the BCO phenotype, Ni et al. (22) reported a pooled prevalence of $54.3 \%$ (ranging from 25.6 to $69 \%$ ) in patients with COPD.

Both asthma and bronchiectasis could incur an exacerbation of the chronic pulmonary disease (AECOPD). The ACO and $\mathrm{BCO}$ phenotypes present common and distinct clinical characteristics and prognoses. In general, the ACO phenotype exhibits a higher eosinophil level and better bronchodilator reversibility, and the BCO phenotype presents more neutrophil and nonreversible characteristics $(23,24)$. Additionally, compared with patients with COPD only, the ACO phenotype

Abbreviations: ABCO, chronic obstructive pulmonary disease patients comorbid with asthma and bronchiectasis; ACO, asthma-chronic obstructive pulmonary disease overlap; ACURE, the acute exacerbation of chronic obstructive pulmonary disease inpatient registry; AECOPD, acute exacerbation of chronic obstructive pulmonary disease; $\mathrm{BCO}$, bronchiectasis- chronic obstructive pulmonary disease overlap; BMI, body mass index; CAT, chronic obstructive pulmonary disease assessment test; CI, confidence interval; COPD, chronic obstructive pulmonary disease; CT, computed tomography; DVT, deep venous thrombosis; FEV1, forced expiratory volume in $1 \mathrm{~s}$; FVC, forced vital capacity; GOLD, the Global Initiative for Chronic Obstructive Lung Disease; HR, hazard ratio; ICU, intensive care unit; $\mathrm{IQR}$, interquartile range; PE, pulmonary embolism; RICU, respiratory intensive care unit; SD, standard deviation; VTE, venous thromboembolism. was characterized with higher probability of exacerbation (5), frequent outpatient and emergency department visits $(25,26)$, but lower rate of hospital readmission (27) and mortality $(28,29)$. The BCO phenotype was associated with increased risk of exacerbation, severe airway obstruction, and higher mortality (30).

Although numerous studies have been conducted, findings were still controversial (1). The notable variation may be due to different study designs, sample sizes, and definitions of diseases used. In addition, most previous data were based on stable stage of COPD, and facts on exacerbation of the disease need to be delineated. In the current manuscript, to address these unanswered questions, we utilized data from an acute exacerbation of COPD inpatient registry (ACURE) to investigate the differences in clinical features and short-term prognosis profiles among Chinese hospitalized patients with AECOPD who were comorbid with asthma and/or bronchiectasis, and those patients without the two comorbidities. We anticipated that our findings could help improve clinical management of the disease in clinical practice.

\section{METHODS}

\section{Study Design and Settings}

Data was retrieved from an acute exacerbation of COPD inpatient registry (ACURE), which was initiated in China to investigate the demographic characteristics, clinical features, diagnoses and treatments, and prognoses among hospitalized patients with COPD who were suffering an acute exacerbation episode (ClinicalTrials.gov registry number: NCT02657525). The ACURE study was started on September 1, 2017 and planned to recruit 7,600 hospitalized patients with AECOPD who were admitted to 161 participating medical centers across China with a maximum of 3-year follow-up. The protocol of the ACURE registry and baseline characteristics of the study population have been published $(31,32)$.

The study protocol, informed consent, and case report form have been approved by the institutional review board at the China-Japan Friendship Hospital (approval number: 201588) and other local participating centers. All the participating patients have provided written informed consent.

\section{Study Population}

The ACURE participants underwent screenings at index hospital admission to confirm their eligibilities for enrollments. Subjects would be enrolled if they fulfilled the following eligibility criteria: 
1) aged 18 years or older; 2) confirmed or suspected to be hospitalized due to AECOPD; 3) not participating in other clinical trial or intervention studies; and 4) agreeing to sign the informed consent. As of February 25, 2020, 4,813 eligible patients met the inclusion and exclusion criteria.

\section{Procedures and Measurements Data Collection}

In the ACURE registry, patient management was at the discretion of clinical physicians. Well-designed and sophisticated questionnaires were administrated to enrolled participants at baseline, i.e., during the index hospitalization, and at planned follow-up visits, i.e., at day 30 ( \pm 2 days), month 6 ( \pm 12 days), month 12 ( \pm 12 days), month 24 ( \pm 12 days), and month 36 ( \pm 12 days), respectively after the index hospital discharge by trained investigators. The questionnaires consisted of contents on basic and demographic information, inclusion and exclusion criteria, current diagnoses (including symptoms and signs), objective examinations [e.g., routine venous blood, lung function, computed tomography (CT), arterial blood gas, electrocardiograph, cardiac color ultrasound, pulmonary ventilation/perfusion image, lower extremity venous ultrasound, and etiological examinations where necessary], history and management of the disease (especially while in the stable condition), predisposing factors and prevention of the exacerbation, pharmacological and nonpharmacological (e.g., respiratory support) treatments in the hospital, cost, outcomes at hospital discharge, and management and outcomes during the follow-ups. Data of any exacerbations that did not occur in the scheduled visits were also collected.

All data were uploaded to an online electronic data capture system. Data quality was regularly monitored by a concerted project and data management team. For instance, missing values, outliers, and illogical information will be sent to the local participating centers for timely amendment.

\section{Diagnoses of Diseases}

In current analyses, spirometric COPD was diagnosed as the presence of a post-bronchodilator forced expiratory volume in $1 \mathrm{~s}$ (FEV1) divided by the forced vital capacity (FVC) with a value of less than 0.70 , which indicated a persistent airflow limitation according to the Global Initiative for Chronic Obstructive Lung Disease (GOLD) 2021 report (10). Asthma was diagnosed by the presence of both variable expiratory airflow limitation and a characteristic pattern of respiratory symptoms for instance wheezing, shortness of breath (dyspnea), chest tightness, and/or cough in adults according to the Global Initiative for Asthma (GINA) 2021 report (9). Bronchiectasis was diagnosed by the presence of both bronchial dilation on CT and clinical symptoms, such as cough, sputum production, and/or recurrent respiratory infection in adults according to the European Respiratory Society (33) and British Thoracic Society guidelines (34). The ACO phenotype was defined as spirometry-diagnosed COPD and asthma. The BCO phenotype was confirmed by spirometric COPD and CTbased bronchiectasis. Patients with COPD both comorbid with asthma and bronchiectasis were termed ABCO phenotype.
Information on disease diagnoses were obtained from patients' medical records during their index hospitalization and critically reviewed by local and central principal investigators/pulmonary physicians. More details on diagnoses of diseases are provided in the Supplementary Material S1.

\section{Variables}

Data on demographic and clinical characteristics, laboratory, lung function, and image (e.g., CT) tests, short- and longterm prognoses were comprehensively collected. Lung function test was performed when the situation of the patient was relatively stable after admission. If multiple lung function tests were conducted, the latest result was chosen before discharge. Bronchiectasis was diagnosed once any of the CT scan result was confirmed during hospitalization. Classification of the severity of airflow limitation was categorized into four grades based on the 2021 GOLD report: GOLD 1 (mild, FEV1 $\geq 80 \%$ predicted), GOLD 2 (moderate, $50 \% \leq \mathrm{FEV} 1<80 \%$ predicted), GOLD 3 (severe, $30 \% \leq \mathrm{FEV} 1<50 \%$ predicted), and GOLD 4 (very severe, FEV $1<30 \%$ predicted) (10).

In this manuscript, short-term clinical outcomes referred to the length of index hospital stay, recurrence of exacerbation, and exacerbation-related hospital readmission within 30 days after the index hospital discharge. Definitions of clinical outcomes are explicated in the Supplementary Material S1.

\section{Statistical Analyses}

Mean and standard deviation (SD) were calculated for normally distributed continuous variables, otherwise median and interquartile range (IQR) were presented for abnormally distributed ones. Frequencies and percentages were calculated for categorical variables. Characteristics of patients within phenotypes were compared with the utilization of Student's $t$-test (normal distributed data, two-group comparison) or Wilcoxon rank-sum test (abnormal distributed data, two-group comparison) or Kruskal-Wallis test (abnormal distributed data, three or above group comparison) as appropriate for continuous variables, and using Pearson's Chi-square test or Fisher's exact test as appropriate for categorical ones.

A multivariable linear regression model was used to assess the associations of predictors with a continuous outcome (i.e., length of hospital stay). Multivariable Cox proportional hazards regression model was adapted to investigate the associations with day 30 outcomes (i.e., recurrence of exacerbation and exacerbation-related hospital readmission). Variables that showed significant associations in univariable analyses $(p<$ 0.10 ), and factors (e.g., age, gender, BMI, smoking status, and FEV1) that previously had been reported to be associated with prognoses of COPD were further adjusted in the multivariable statistical models (35). A stepwise selection scheme with an entry level of 0.10 and a stay level of 0.05 was applied. Multicollinearity diagnosis was performed before conducting the multivariable analyses. Details on multivariable models employed for stepwise selection of independent predictors are given in the Supplementary Material S2. Kaplan-Meier curve and log-rank test were utilized to determine the differences in day 30 outcomes between phenotypes. 
Statistical significance was defined as achieving a two-sided $P$ value of less than 0.05 . Statistical analyses were performed using Statistical Analysis System (version 9.4) and R Project (version 4.0.5).

\section{RESULTS}

Of the overall 4,813 eligible patients with AECOPD, 63 (1.31\%) patients were comorbid both with asthma and bronchiectasis (ABCO), 338 (7.02\%) and $492(10.22 \%)$ patients were identified as ACO and BCO phenotypes, respectively, and 3,920 (81.45\%) patients did not coexist with asthma or bronchiectasis (Figure 1).

Demographic and clinical characteristics among the ACO, $\mathrm{BCO}$, and $\mathrm{ABCO}$ phenotypes as well as those patients without asthma or bronchiectasis are shown in Table 1. Relatively, the ABCO phenotype patients had a younger age with a median of 62.99 years (IQR: 55.93-69.48), and the patients without asthma or bronchiectasis had an older age with a median of 70.15 years (IQR: 64.37-76.82). The BCO phenotype patients and patients without asthma or bronchiectasis were similar in BMI with a median of $21.79 \mathrm{~kg} / \mathrm{m}^{2}$ (IQR: 19.47-23.97) and $21.79 \mathrm{~kg} / \mathrm{m}^{2}$ (IQR: $19.49-24.22$ ), respectively. In addition, patients without asthma or bronchiectasis were more of the male gender (79.90\%) and smokers (71.12\%) with a longer history of smoking (median: 32.45 years, IQR: 0.00-43.91). The ACO and
ABCO phenotype patients suffered more prior allergic episodes with a proportion of 18.05 and $19.05 \%$, respectively. The ACO phenotype patients exhibited higher level of eosinophil and better lung reversibility. The four phenotype patients showed no significant difference in all-cause mortality, intensive care unit (ICU)/respiratory intensive care unit (RICU) admission, length of stay, and COPD assessment test (CAT) score change in the index hospitalization, and neither in the day 30 outcomes, i.e., all-cause mortality, recurrence of exacerbation, and all-cause and exacerbation-related hospital readmission (Table 1).

Independent predictors associated with hospitalized and day30 outcomes across the four phenotypes are shown in Figure 2. Varied factors were found in predicting the length of hospital stay among different phenotypes. For example, cancer was solely the strong predictor within the ABCO phenotype patients. Peptic ulcer, venous thromboembolism (VTE), and $\mathrm{PaCO}_{2}$ were the independent influencing factors in the BCO phenotype patients. More predicting factors such as age, education level, vaccination within the past 5 years, hospitalization due to AECOPD in the prior year, level of white blood cell, VTE, pulmonary interstitial fibrosis, failure of respiration, etc. were included in the profiles in the patients without asthma or bronchiectasis (Figure 2).

The predicting profiles of day 30 outcomes also exhibited distinct features. Community-acquired pneumonia, cancer, and the level of eosinophils were independent predictors of exacerbation recurrence in $\mathrm{ACO}$ phenotype patients. In $\mathrm{BCO}$

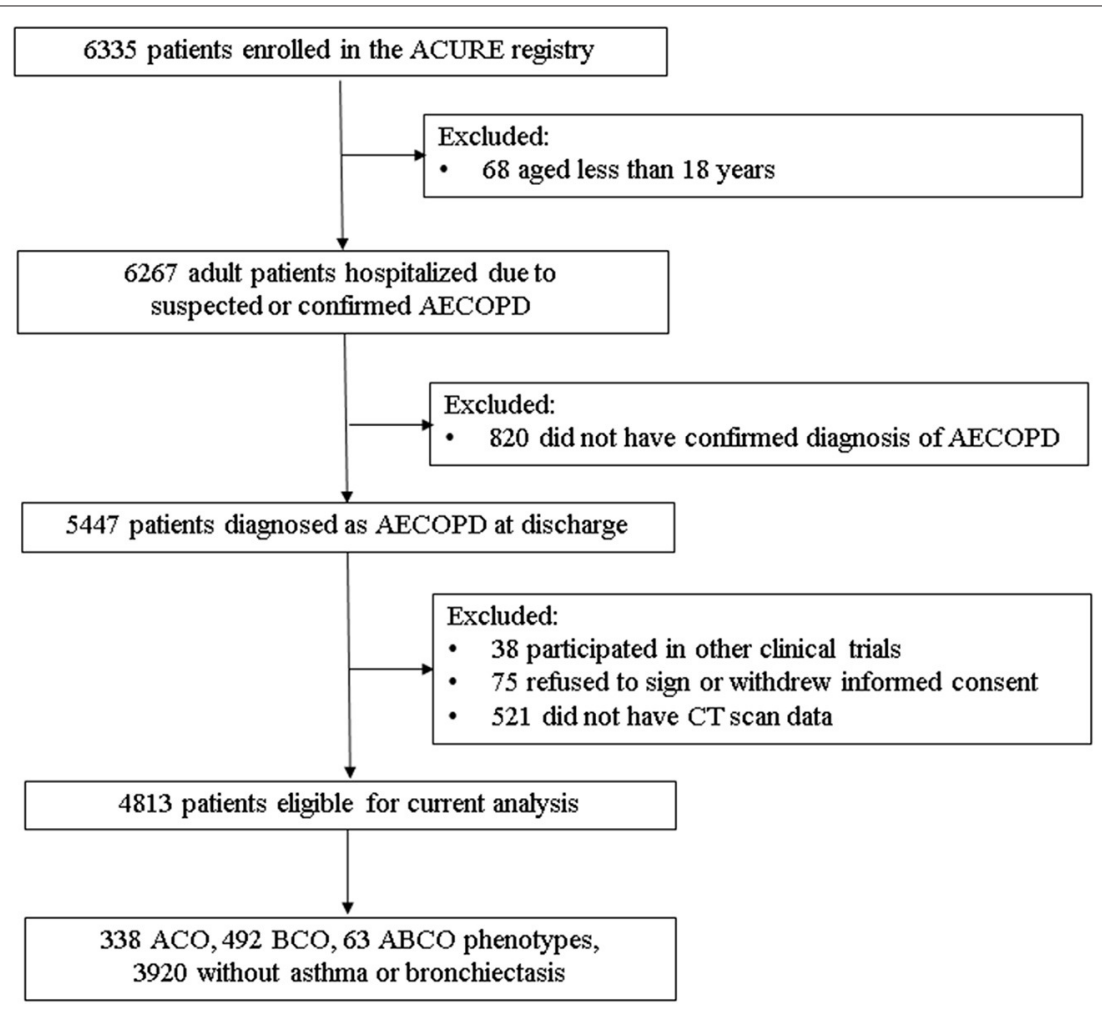

FIGURE 1 | Flowchart of diagram in current analyses. ABCO, chronic obstructive pulmonary disease patients comorbid with asthma and bronchiectasis; ACO, asthma-chronic obstructive pulmonary disease overlap; ACURE, the acute exacerbation of chronic obstructive pulmonary disease inpatient registry; AECOPD, acute exacerbation of chronic obstructive pulmonary disease; BCO, bronchiectasis- chronic obstructive pulmonary disease overlap; CT, computed tomography. 
TABLE 1 | Main characteristics and comparisons among ACO, BCO, ABCO phenotypes, and those without asthma or bronchiectasis in hospitalized AECOPD patients.

\begin{tabular}{|c|c|c|c|c|c|c|c|}
\hline Main variables & All patients $(N=4,813)$ & ACO phenotype $(N=338)$ & $\begin{array}{l}\text { BCO phenotype ( } N \\
=492)\end{array}$ & $\begin{array}{l}\text { ABCO phenotype } \\
\qquad(N=63)\end{array}$ & $\begin{array}{c}\text { Patients without asthma } \\
\text { or bronchiectasis }(N= \\
3,920)\end{array}$ & $\begin{array}{c}P \text { value for } \\
\text { ACO vs. BCO }\end{array}$ & $\begin{array}{c}P \text { value among } \\
\text { four } \\
\text { phenotypes }\end{array}$ \\
\hline
\end{tabular}

\section{Demographics}

Age (years), median (IQR)

$69.70(63.72,76.46)$

$66.19(59.63,73.53)$

$69.14(63.16,75.44) \quad 62.99(55.93,69.48)$

$70.15(64.37,76.82)$

$<0.0001$

$<0.0001$

Male

Female

BMI $\left(\mathrm{kg} / \mathrm{m}^{2}\right)$, median (IQR)

$3,751(77.93)$

$1,062(22.07)$

$21.97(19.53,24.24)$

$232(68.64)$
$106(31.36)$

342 (69.51)

$45(71.43)$

$150(30.49)$

$18(28.57)$

$23.41(20.57,25.71)$

$21.79(19.47,23.97) \quad 23.18(20.20,26.04)$

$788(20.10)$

Education level, $n$ (\%)

Primary school or below

Junior high school

Senior high school

Undergraduate or above

Smoking status, $n(\%)$

Former

Current

Never

Smoking years (years), median (IQR)

\section{Medical history}

Previous allergic episode, $\mathrm{n}(\%)$

Vaccination within past 5 years, $n$ (\%)

Emergency visit due to AECOPD in

prior year (times), median (IQR)

Hospitalization due to AECOPD in

prior year (times), median (IQR)

\section{Other comorbidities, $\mathbf{n}(\%)$}

Respiratory disease

Community-acquired pneumonia

VTE (including PE and DVT)

Pulmonary interstitial fibrosis

Pulmonary heart disease

Failure of respiration

2,372 (49.28)

$155(45.86)$

$238(48.37)$

,570 (32.62)

676 (14.05)

$195(4.05)$

$107(31.66)$

57 (16.86)

2,117 (43.99)

$1,165(24.21)$

$1,531(31.81)$

$30.71(0.00,42.48)$

$19(5.62)$

$177(35.98)$

64 (13.01)

$23(36.51)$

$13(2.64)$

23 (36.51)

$14(22.22)$

114 (33.73)

83 (24.56)

$181(36.79)$

$80(16.26)$

25 (39.68)

141 (41.72)

231 (46.95)

$22.50(0.00,40.00)$

$10.00(0.00,40.00)$

$20.00(0.00,33.00)$

\section{1 (18.05)}

$11(3.25)$

137 (2.85)

$0.00(0.00,1.00)$

$0.00(0.00,1.00)$

55 (11.18)

$10(2.03)$

$0.00(0.00,2.00)$

12 (19.05)

$2(3.17)$

$1.00(0.00,2.00)$

$0.00(0.00,1.00)$

$1.00(0.00,2.00)$

$0.00(0.00,1.00)$

$1.00(0.00,2.00)$

$21.79(19.49,24.22)$

$<0.0001$

$<0.000$

1,956 (49.90)

$1,263(32.22)$

541 (13.80)

$160(4.08)$

1,797 (45.84)

991 (25.28)

$1,132(28.88)$

$32.45(0.00,43.91)$

0.2317

$<0.0001$

427 (10.89)

114 (2.91)

$0.00(0.00,1.00)$

0.0050

0.2707

0.4031

0.6914

0.4868

$1.00(0.00,2.00)$

0.0062

0.0279

$\begin{array}{cc}1,483(30.81) & 103(30.47) \\ 43(0.89) & 5(1.48) \\ 121(2.51) & 10(2.96) \\ 1,015(21.09) & 52(15.38) \\ 1,209(25.12) & 73(21.60) \\ 861(17.89) & \\ 21(0.44) & 59(17.46) \\ 277(5.76) & 0(0.00) \\ & 22(6.51)\end{array}$

$152(30.89)$

$19(30.16)$

$2(0.41)$

$0(0.00)$

$11(2.24)$

2 (3.17)

102 (20.73)

14 (22.22)

131 (26.63)

24 (38.10)

91 (18.50)

$6(9.52)$

5 (1.02)

$0(0.00)$

25 (5.08)

3 (4.76)

$12(2.44)$

$3(4.76)$

1,209 (30.84)

0.8972

0.9984

$36(0.92)$

0.1277

0.5147

0.4201

$98(2.50)$

0.0515

981 (25.03)

0.0983

0.0621

0.0371

705 (17.98)

0.7020

0.3629

$16(0.41)$

0.0836

0.1674

Digestive disease

Gastroesophageal reflux disease

$91(1.89)$

$12(3.55)$

$227(5.79)$

0.3820

0.8279

0.0184 


\section{Main variables}

All patients $(N=4,813) \quad$ ACO phenotype $(N=338)$

BCO phenotype ( $N$

$$
=492)
$$

ABCO

$(N=63)$
phenotype

Peptic ulcer

Other condition

Cancer

Cerebrovascular disease

Peripheral biomarkers, median

(IQR)

White blood cell ( $\left.{ }^{*} 10^{9} / \mathrm{L}\right)$

Platelet $\left({ }^{*} 10^{9} / \mathrm{L}\right)$

Neutrophil (\%)

Neutrophil $\left({ }^{*} 10^{9} / \mathrm{L}\right)$

Lymphocyte (\%)

Lymphocyte $\left({ }^{*} 10^{9} / \mathrm{L}\right)$

Eosinophil (\%)

Eosinophil ( $\left.{ }^{*} 10^{9} / L\right)$

Direct bilirubin (umol/L)

Alkaline phosphatase (U/L)

Gamma glutathione transpeptidase (U/L)

\section{Arterial blood gas, median (IQR)}

$\mathrm{PaCO}_{2}(\mathrm{mmHg})$

$\mathrm{PaO}_{2}(\mathrm{mmHg})$

$\mathrm{SaO}_{2}(\%)$

\section{Lung function test}

Post-bronchodilator lung function

median (IQR)

FEV1 (L)

FVC (L)

FEV1/FVC (\%)

FEV1 \% predicted value (\%)

FVC \% predicted value (\%)

GOLD categories, $n$ (\%)

GOLD 1

GOLD 2

GOLD 3

GOLD 4

Short-term clinical outcomes

Within the index hospitalization

$69(1.43)$
$55(1.14)$
$230(4.78)$

$7.18(5.57,9.40)$
$0.02(0.02,0.03)$
$.90(55.50,77.60)$
$5.02(3.54,7.20)$
$6.22(7.90,24.40)$
$1.27(0.86,1.75)$
$1.10(0.10,2.70)$
$0.10(0.02,0.21)$
$3.20(1.90,4.70)$
$0.01(0.01,0.01)$
$0.00(0.00,8.80)$

$34.30(0.00,43.40)$

$0.00(0.00,68.90)$

$0.00(0.00,94.80)$

$0.93(0.65,1.32)$

$1.97(1.45,2.54)$

$0.50(0.42,0.59)$

$0.40(0.28,0.56)$

$0.65(0.50,0.82)$

\begin{abstract}
246 (5.11)
$1,102(22.90)$

1,490 (30.96)

$1,185(24.62)$
\end{abstract}

$6(1.22)$

5 (1.02)

$22(4.47)$

22 (6.51)

$7.42(5.70,9.38)$

$0.02(0.02,0.03)$

$64.10(53.30,73.70)$

$4.73(3.30,6.93)$

$18.40(7.80,26.60)$

$1.40(0.94,1.94)$

$1.40(0.10,4.10)$

$0.12(0.03,0.33)$

$3.10(1.90,4.40)$

$0.01(0.01,0.02)$

$0.00(0.00,14.66)$

$33.10(0.00,41.20)$

$0.00(0.00,64.40)$

$0.00(0.00,94.80)$

$1.20(0.87,1.57)$

$2.35(1.77,2.94)$

$0.54(0.44,0.61)$

$0.50(0.36,0.64)$

$0.77(0.61,0.90)$

$23(6.80)$

121 (35.80)

102 (30.18)

44 (13.02)

$\begin{array}{cc}7.48(5.76,10.10) & 7.71(6.13,10.27) \\ 0.02(0.02,0.03) & 0.02(0.02,0.02) \\ 70.00(58.45,79.10) & 67.10(57.30,77.50) \\ 5.34(3.77,7.86) & 5.72(4.60,7.22) \\ 15.10(8.40,23.20) & 16.70(7.20,24.20) \\ 1.23(0.87,1.70) & 1.54(1.10,2.19) \\ 0.90(0.10,2.60) & 0.90(0.02,2.30) \\ 0.09(0.02,0.20) & 0.11(0.04,0.27) \\ 3.10(1.87,4.60) & 3.20(2.20,4.10) \\ 0.01(0.01,0.01) & 0.01(0.01,0.01) \\ 0.00(0.00,0.02) & 0.00(0.00,15.30)\end{array}$

$32.60(0.00,43.60)$

$0.00(0.00,65.25)$

$0.00(0.00,93.80)$

$0.00(0.00,40.90)$

$0.00(0.00,0.00)$

$0.00(0.00,84.40)$

$0.87(0.62,1.17)$

$1.79(1.33,2.27)$

$0.51(0.43,0.58)$

$0.38(0.28,0.51)$

$0.62(0.48,0.76)$

\begin{abstract}
$1.00(0.74,1.32)$
$2.21(1.73,2.81)$

$0.44(0.40,0.52)$

$0.39(0.33,0.51)$

$0.67(0.59,0.79)$
\end{abstract}

Patients without asthma

or bronchie

3,920)

58 (1.48)

$45(1.15)$

184 (4.69)

$7.11(5.51,9.30)$

$0.02(0.02,0.03)$

$67.90(55.45,77.67)$

$4.97(3.50,7.19)$

$16.21(7.80,24.30)$

$1.26(0.85,1.73)$

$1.10(0.10,2.70)$

$0.10(0.02,0.21)$

$3.20(1.90,4.70)$

$0.01(0.01,0.01)$

$0.00(0.00,7.80)$

$34.70(0.00,43.50)$

$0.00(0.00,69.90)$

$68.30(0.00,94.90)$

$0.91(0.64,1.31)$

$1.95(1.43,2.53)$

$0.50(0.42,0.59)$

$0.39(0.27,0.56)$

$0.65(0.49,0.82)$

$1(1.59)$
$12(19.05)$
$26(41.27)$
$12(19.05)$

200 (5.10)

879 (22.42)

$1,184(30.20)$

1,008 (25.71)
$P$ value for

ACO vs. BCO

$P$ value among

four

phenotypes

0.4442




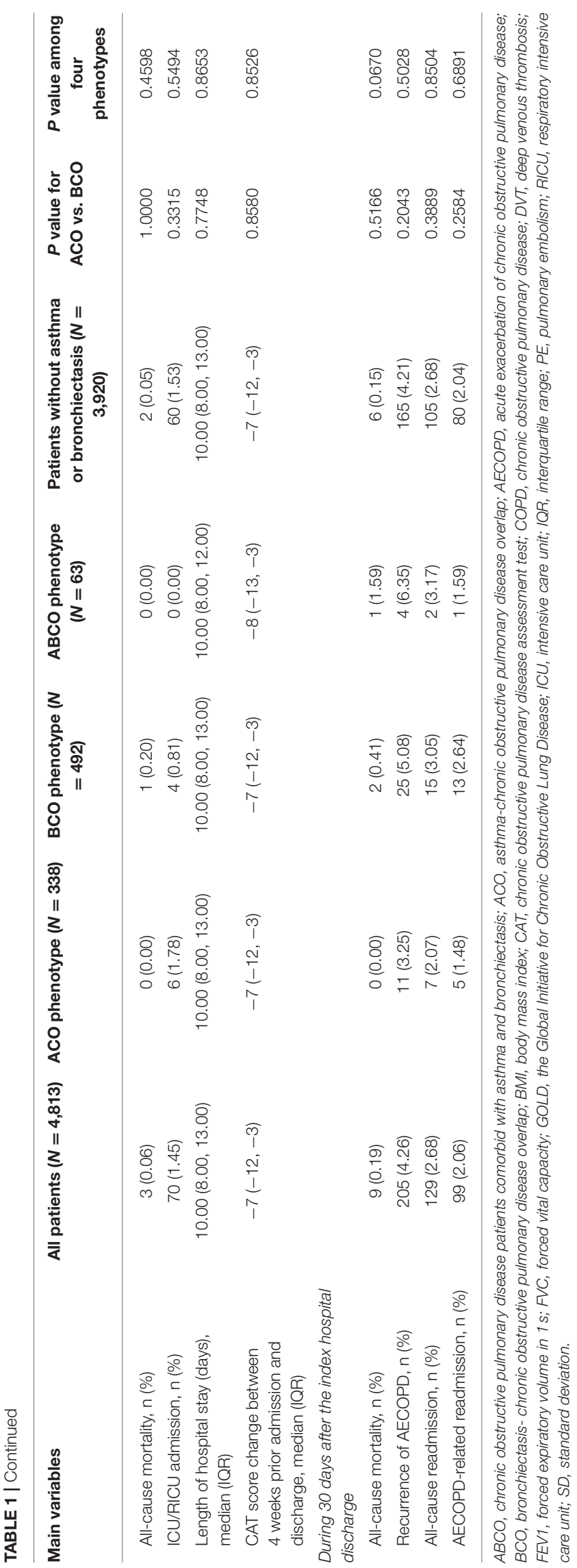

phenotype patients, BMI, gastroesophageal reflux disease, and level of alkaline phosphatase were associated with increased probabilities of exacerbation recurrence. Previous allergic episode, pulmonary heart disease, levels of white blood cells, and gamma glutathione transpeptidase were independent predictors in the patients without asthma or bronchiectasis. With respect to the exacerbation-related readmission, acute heart failure was the single strong predictor in the $\mathrm{BCO}$ phenotype patients. Times of hospitalization and emergency visit due to exacerbation in the prior year, levels of white blood cells and platelets, and percentage of neutrophils were positively associated with elevated exacerbation-related readmission rate in patients without asthma or bronchiectasis (Figure 2).

Kaplan-Meier curves and log-rank tests for recurrence of exacerbation and exacerbation-related readmission within 30 days after the index hospital discharge between ACO and BCO phenotype patients, between ACO or BCO phenotype patients and patients without asthma or bronchiectasis, and among the four phenotype patients are shown in Figure 3, respectively. Significant differences were not seen among these phenotypes.

\section{DISCUSSION}

In current manuscript, clinical characteristics and short-term prognosis profiles of hospitalized patients with COPD due to exacerbation comorbid with asthma and/or bronchiectasis, as well as those patients without the two comorbidities were fully analyzed. The prevalence of $\mathrm{ACO}, \mathrm{BCO}$, and $\mathrm{ABCO}$ phenotypes were $7.02,10.22$, and $1.31 \%$, respectively.

Major findings of our analyses confirmed that patients with COPD comorbid with asthma and/or bronchiectasis or without showed distinct clinical features, particularly in age, gender, BMI, smoking status, prior allergic and COPD history, hospitalization due to exacerbation in the past year, comorbidities (e.g., pulmonary artery hypertension, lung cancer, failure of respiration, diabetes, gastroesophageal reflux disease, and anxiety or depression), levels of peripheral biomarkers (e.g., neutrophil, lymphocyte, eosinophil, creatinine, highsensitivity C-reactive protein, fibrinogen, and $\mathrm{N}$-terminal probrain natriuretic peptide), and lung function. Differences between $\mathrm{ACO}$ and $\mathrm{BCO}$ phenotypes were similar to previous research $(16,24,27)$, but characteristics of $\mathrm{ABCO}$ phenotype were seldom described $(23,24)$. In our data, compared with $\mathrm{ACO}$ and $\mathrm{BCO}$ phenotype patients, the $\mathrm{ABCO}$ phenotype patients had significantly younger age, experienced more prior allergic episodes, and COPD diagnoses, but had a shorter COPD history, comorbid with more failure of respiration and anxiety or depression. Although demographic and clinical characteristics varied between the four phenotypes of patients, no statistical differences were observed with the short-term outcomes. The findings need to be validated using a larger and an independent sample as well as with longer follow-up information.

The prevalences of ACO and BCO phenotypes in our study were lower than previous reports $(17-22,36)$, maybe due to varied study populations, actual lower prevalence, different diagnosis criteria used, and underestimation of the diseases. 


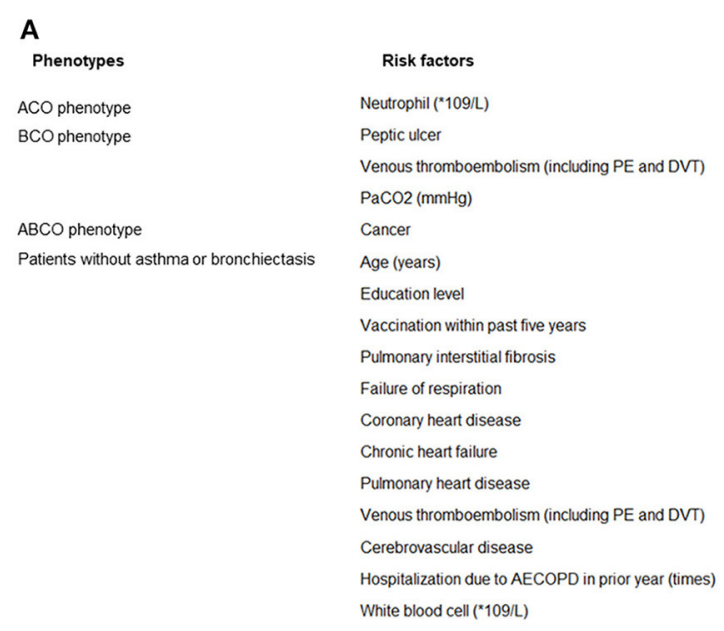

B

Gamma glutathione transpeptidase (U/L)

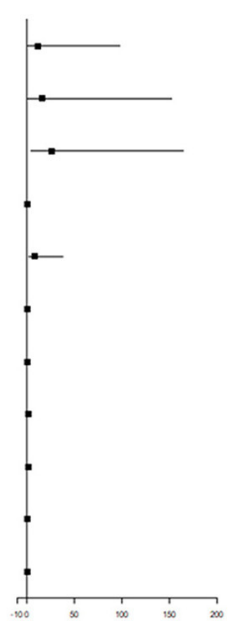

Direct bilirubin (umoVL)

\section{$\beta(95 \% \mathrm{Cl})$}

$0.285(0.062-0.508)$

$8.089(4.193-11.984)$

$8.667(3.161-14.173)$

$0.024(0.004-0.044)$

$19.745(14.110-25.379)$

$0.023(0.002-0.043)$

$0.276(0.066-0.486)$

$1.634(0.675-2.592)$

$1.909(0.793-3.025)$

$0.764(0.342-1.187)$

$0.918(0.455-1.381)$

$0.822(0.057-1.586)$

0.810 (0.365-1.255)

$2.311(0.260-4.363)$

0.854 (0.036-1.673)

0.241 (0.095-0.388)

$0.066(0.021-0.110)$
P value

0.0125

$<.0001$

0.0022

0.0191

$<.0001$

0.0321

0.0099

0.0008

0.0008

0.0004

0.0001

0.0352

0.0004

0.0273

0.0409

0.0013

0.0038

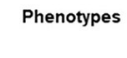

ACO phenotype

BCO phenotype

Patients without asthma or bronchiectasis

\section{Previous allergic episode}

Pulmonary heart disease

White blood cell ( $\left.{ }^{*} 109 / L\right)$

\section{Risk factors}

Community-acquired pneumonia

Cancer

Eosinophil (*109/L)

BMI (kg/m2)

Gastroesophageal reflux disease

Alkaline phosphatase (U/L)

de

C

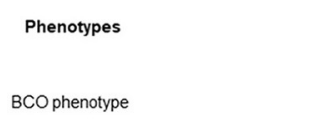

Patients without asthma or bronchiectasis

\section{Risk factors \\ Acure heart failure}

Hospitalization due to AECOPD in prior year (times)

Emergency visit due to AECOPD in prior year (times)

White blood cell ("109/L)

Platelet (“109/L)

Neutrophil (\%)

$\begin{array}{cc}\text { HR }(95 \% \mathrm{Cl}) & \text { P value } \\ 12.227(1.528-97.831) & 0.0183 \\ 16.120(1.706-152.346) & 0.0153 \\ 26.187(4.166-164.586) & 0.0005 \\ 1.122(1.054-1.194) & 0.0003 \\ 8.359(1.847-37.827) & 0.0058 \\ 0.680(0.513-0.902) & 0.0075 \\ 1.015(1.006-1.025) & 0.0017 \\ 1.695(1.032-2.784) & 0.0371 \\ 1.968(1.335-2.899) & 0.0006 \\ 1.073(1.031-1.117) & 0.0005 \\ 1.004(1.001-1.007) & 0.0106 \\ \end{array}$

$24.395(2.988-199.175) \quad 0.0029$

$1.229(1.048-1.440) \quad 0.0110$

$1.194(1.019-1.398) \quad 0.0285$

$1.068(1.007-1.131) \quad 0.0272$

$1.004(1.000-1.008) \quad 0.0473$

$1.014(1.001-1.026) \quad 0.0279$

FIGURE 2 | (A) Independent risk factors associated with length of index hospital stay. (B) Independent risk factors associated with recurrence of AECOPD within 30 days after the index hospital discharge. (C) Independent risk factors associated with exacerbation-related hospital readmission within 30 days after the index hospital discharge. $\mathrm{ABCO}$, chronic obstructive pulmonary disease patients comorbid with asthma and bronchiectasis; ACO, asthma-chronic obstructive pulmonary disease overlap; AECOPD, acute exacerbation of chronic obstructive pulmonary disease; $\mathrm{BCO}$, bronchiectasis-chronic obstructive pulmonary disease overlap; BMI, body mass index; $\mathrm{Cl}$, confidence interval; DVT, deep venous thrombosis; HR, hazard ratio; ICU, intensive care unit; PE, pulmonary embolism; RICU, respiratory intensive care unit. 
A

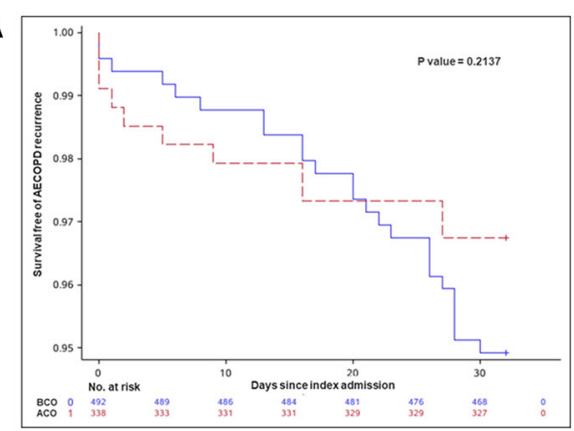

(a) Recurrence of AECOPD

B

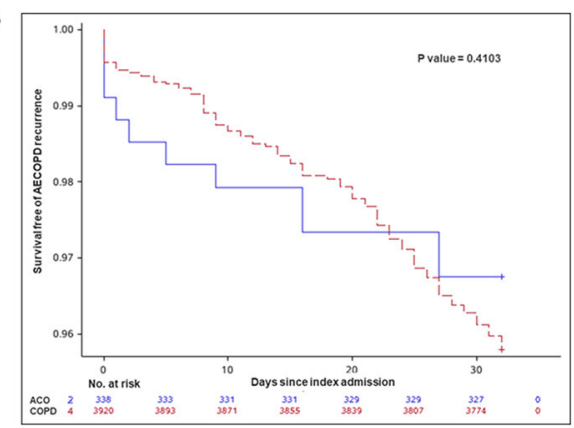

(a) Recurrence of AECOPD

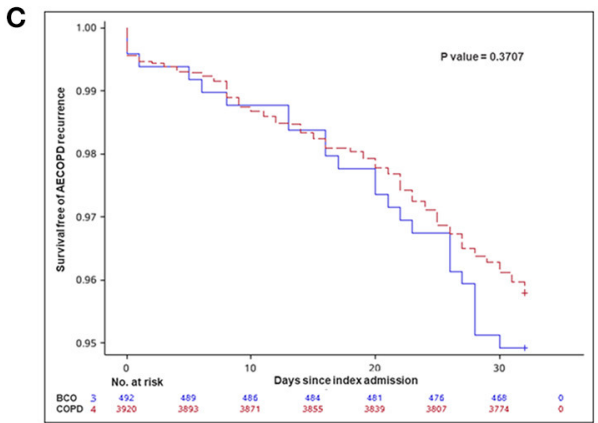

(a) Recurrence of AECOPD

D

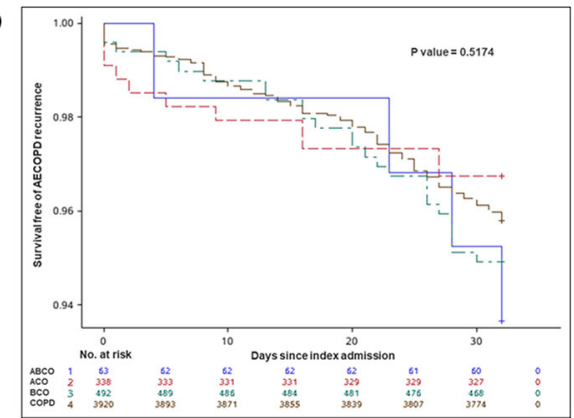

(a) Recurrence of AECOPD

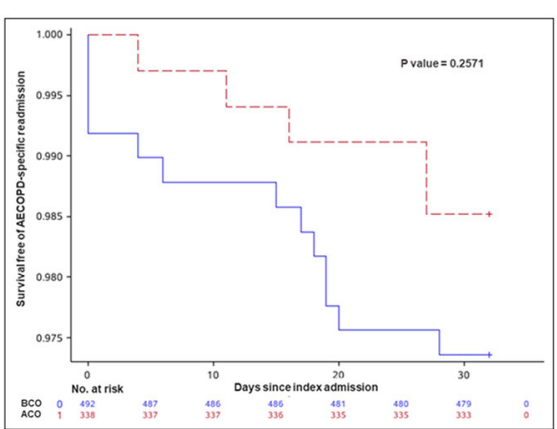

(b) AECOPD-related readmission

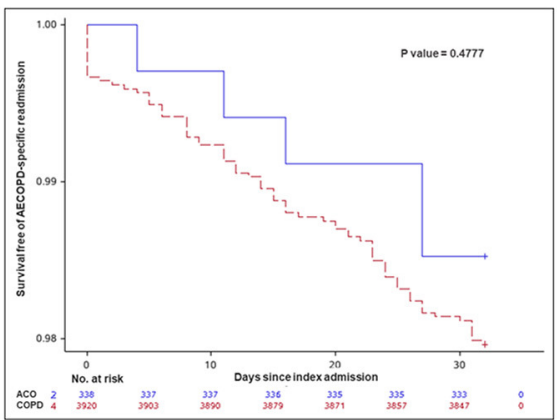

(b) AECOPD-related readmission

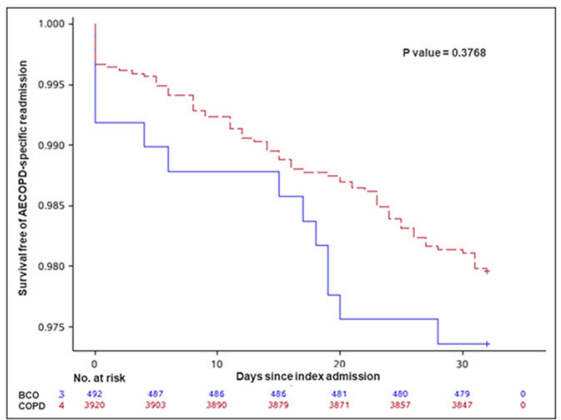

(b) AECOPD-related readmission

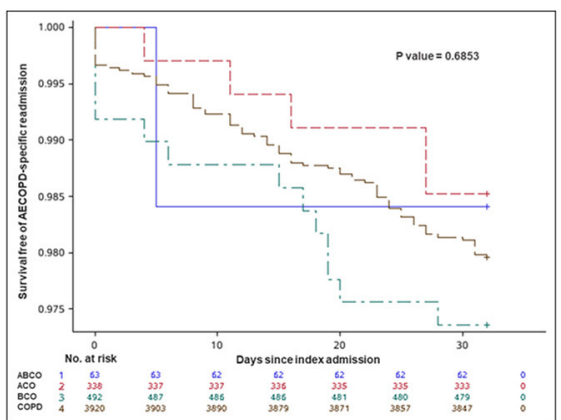

(b) AECOPD-related readmission

FIGURE 3 | (A) Kaplan-Meier curve and log-rank test for day 30 outcomes between ACO and BCO phenotypes in hospitalized patients with AECOPD. Kaplan-Meier curve and log-rank test for day 30 outcomes (a) recurrent exacerbation $(P=0.2137)$ and $(b)$ exacerbation-related readmission $(p=0.2571)$ between $\mathrm{ACO}$ and $\mathrm{BCO}$ phenotypes in hospitalized patients with AECOPD. (B) Kaplan-Meier curve and log-rank test for day 30 outcomes between ACO phenotype and patients without

(Continued) 
FIGURE 3 | asthma or bronchiectasis in hospitalized patients with AECOPD. Kaplan-Meier curve and log-rank test for day 30 outcomes (a) recurrent exacerbation (p $=0.4103)$ and $(b)$ exacerbation-related readmission $(p=0.4777)$ between ACO phenotype and patients without asthma or bronchiectasis in hospitalized patients with AECOPD. (C) Kaplan-Meier curve and log-rank test for day 30 outcomes between BCO phenotype and patients without asthma or bronchiectasis in hospitalized patients with AECOPD. Kaplan-Meier curve and log-rank test for day 30 outcomes (a) recurrent exacerbation $(P=0.3707)$ and (b) exacerbation-related readmission ( $P=0.3768$ ) between BCO phenotype and patients without asthma or bronchiectasis in hospitalized patients with AECOPD. (D) Kaplan-Meier curve and log-rank test for day 30 outcomes among four phenotypes in hospitalized patients with AECOPD. Kaplan-Meier curve and log-rank test for day 30 outcomes (a) recurrent exacerbation $(P=0.5174)$ and $(b)$ exacerbation-related readmission $(P=0.6853)$ among four phenotypes in hospitalized patients with AECOPD. ABCO, chronic obstructive pulmonary disease patients comorbid with asthma and bronchiectasis; ACO, asthma-chronic obstructive pulmonary disease overlap; AECOPD, acute exacerbation of chronic obstructive pulmonary disease; BCO, bronchiectasis-chronic obstructive pulmonary disease overlap; COPD, chronic obstructive pulmonary disease.

CT scans for bronchiectasis and difficulties with diagnosing asthma clinically could also result in misclassification bias while the phenotypes were categorized. Additionally, differences in genetic backgrounds, allergens, chemical exposures, and dietary dissimilarities from other parts of the world may be alternative explanations, and if demography would play any role among Chinese patients living in other continents needs to be investigated. The latter assumptions should be tested via international multicenter collaboration between countries and ethnicities.

Furthermore, the entity of ACO phenotype has been debated for years, and the diagnosis criteria are developing. Cosio BG et al. used a composite criteria to define ACO, which included positive bronchodilator response, medical history of asthma, and higher levels of blood eosinophil and IgE (37). Hansen JE et al. suggested adopting a new bronchodilator response grading strategy based on FEV1 and FVC to identify the distinct ACO phenotype (38) and Fortis et al. (39) found that combined FEV1 and FVC bronchodilator response could be more sensitive to indicate an ACO phenotype, based on the observation that patients with asthma may have a greater bronchodilator response than those with COPD only. However, whether the bronchodilator response has diagnostic value in separating COPD and asthma is doubted (40). Validation studies are needed and consensus has to be achieved.

Current analyses were based on the largest ongoing multicenter registry on hospitalized patients with AECOPD in China. Characteristics and short-term prognoses of common but distinct phenotypes of COPD, i.e., ACO, BCO, ABCO phenotypes and those patients without the two diseases, were fully described. The findings of current analyses could provide the real-world evidence, as well as hints for disease management and further research. Meanwhile, several limitations of current analyses should be stated. First, some estimations of the associations lacked precision, i.e., a broad confidence interval might be due to the limited number of patients or events. Second, some potential impact factors and outcomes were not considered to avoid amplification of multiple testing and the type I error. Third, data on other common phenotypes such as chronic bronchitis and emphysema were not included. Additionally, some factors that would have influences on outcomes of interests were not collected, such as secondhand smoking, biomass fuel exposure, outdoor air pollution, etc, and alternative clinical outcomes including composite ones should be considered. With the ongoing recruitment and follow-up of the ACURE registry, a larger number of patients and long-term prognosis analyses in the future are possible.

\section{CONCLUSIONS}

Current findings revealed that ACO, BCO, their overlaps (ABCO), and those patients without the two comorbidities had distinct clinical features, but did not differ in short-term prognoses. Further replication and validation in a larger and independent sample are warranted.

\section{DATA AVAILABILITY STATEMENT}

The raw data supporting the conclusions of this article will be made available from the corresponding authors on reasonable request, without undue reservation.

\section{ETHICS STATEMENT}

The study involving human participants was reviewed and approved by the Institutional Review Board of the ChinaJapan Friendship Hospital (approval number: 2015-88). The participants provided their written informed consent to participate in this study.

\section{THE CHINA ACUTE EXACERBATION OF CHRONIC OBSTRUCTIVE PULMONARY DISEASE INPATIENT REGISTRY (ACURE) INVESTIGATORS}

We deeply appreciated continuous supports and contributions from the following 161 hospitals and the local investigators: The First Affiliated Hospital of Hunan University of Medicine, Hunan Province; The First People's Hospital of Huaihua City, Hunan Province; Affiliated Hospital of Inner Mongolia University for the Nationalities, Inner Mongolia Autonomous Region; The People's Hospital of the Xishuangbanna Dai Nationality Autonomous Prefecture, Yunnan Province; Tongji Hospital, Tongji Medical College, Huazhong University of Science \& Technology, Hubei Province; Zhangjiagang First People's Hospital, Jiangsu Province; The First Affiliated Hospital of Zhengzhou University, Henan Province; First Hospital of Shanxi Medical University, Shanxi Province; Central Hospital Affiliated to ShenYang Medical 
College, Liaoning Province; Liuzhou Workers Hospital, Guangxi Zhuang Autonomous Region; LongHua Hospital Shanghai University of Traditional Chinese Medicine, Shanghai City; The First Affiliated Hospital of Guangzhou University of Chinese Medicine, Guangdong Province; People's Hospital of Ji'an County, Jiangxi Province; Gongzhuling Central Hospital, Jilin Province; Chongqing Sixth People's Hospital, Chongqing City; Shanghai Putuo District Central Hospital (Putuo Hospital Affiliated to Shanghai University of Traditional Chinese Medicine), Shanghai City; The Second Affiliated Hospital of Tianjin University of Traditional Chinese Medicine, Tianjin City; Shanxi Fenyang Hospital, Shanxi Province; Taihe Hospital (Affiliated Taihe Hospital of Hubei University of Medicine), Hubei Province; Ulanqab Second Hospital, Inner Mongolia Autonomous Region; Nanjing Pukou District Central Hospital (Pukou Branch of Jiangsu Provincial People's Hospital), Jiangsu Province; Benxi Central Hospital, Liaoning Province; The Third People's Hospital of Jingdezhen, Jiangxi Province; Chongqing Jiulongpo District First People's Hospital, Chongqing City; Second Hospital of Shanxi Medical University, Shanxi Province; Ulanqab Central Hospital, Inner Mongolia Autonomous Region; Mianyang Central Hospital, Sichuan Province; Suining Central Hospital, Sichuan Province; The People's Hospital of Qitaihe, Heilongjiang Province; Affiliated Hospital of Zunyi Medical University, Guizhou Province; Inner Mongolia Baogang Hospital, Inner Mongolia Autonomous Region; Qiqihar Traditional Chinese Medicine Hospital, Heilongjiang Province; The Second Xiangya Hospital of Central South University, Hunan Province; Chengdu Qingbaijiang District People's Hospital, Sichuan Province; Minority Hospital of Guangxi Zhuang Autonomous Region (Minority Hospital Affiliated to Guangxi Medical University), Guangxi Zhuang Autonomous Region; Meihekou Central Hospital, Jilin Province; The People's Hospital of Yi County, Liaoning Province; The Central Hospital of Yongzhou, Hunan Province; Fujian Provincial Hospital, Fujian Province; The First Affiliated Hospital of Guiyang College of Traditional Chinese Medicine, Guizhou Province; The People's Hospital of Wanzhou District, Chongqing City; Renhe Hospital, Baoshan District, Shanghai City; The People's Hospital of Nanchuan, Chongqing City; Miyun District Hospital, Beijing City; The First People's Hospital of Chuzhou, Anhui Province; Shuguang Hospital Affiliated to Shanghai University of Traditional Chinese Medicine, Shanghai City; The Second Affiliated Hospital of Guangxi Medical University, Guangxi Zhuang Autonomous Region; Chengdu Second People's Hospital, Sichuan Province; 903 Hospital, Sichuan Province; The People's Hospital of Gaozhou, Guangdong Province; The Fourth Affiliated Hospital of Anhui Medical University, Anhui Province; Henan Provincial People's Hospital, Henan Province; The Second Affiliated Hospital of Shandong University of Traditional Chinese Medicine, Shandong Province; The Affiliated Hospital of Southwest Medical University, Sichuan Province; The Second People's Hospital of Guiyang, Guizhou Province; The Second Affiliated Hospital of Guilin Medical University, Guangxi Zhuang Autonomous Region; Inner Mongolia Xing'an League People's Hospital, Inner Mongolia Autonomous Region; Affiliated Hospital of Jiangxi University of Traditional Chinese Medicine, Jiangxi Province; The First Hospital of Kunming, Yunnan Province; The People's Hospital of Langfang City, Hebei Province; Shanghai Traditional Chinese Medicine-Integrated Hospital, Shanghai City; The First Hospital of Changsha, Hunan Province; The Fifth People's Hospital of Datong, Shanxi Province; Yichang Central People's Hospital, Hubei Province; Hebei Provincial Hospital of Traditional Chinese Medicine, Hebei Province; Fuling Central Hospital, Chongqing City; Jiangxi Pingxiang People's Hospital, Jiangxi Province; Shanxi Bethune Hospital (Shanxi Academy of Medical Sciences), Shanxi Province; Ths People's Hospital of Maoming, Guangdong Province; People's Hospital of Anshun City, Guizhou Province; China Resources WISCO General Hospital, Hubei Province; Qinghai Provincial People's Hospital, Qinghai Province; Shanghai Dongfang Hospital (Dongfang Hospital affiliated to Tongji University), Shanghai City; Xinjiang Uygur Autonomous Region People's Hospital, Xinjiang Uygur Autonomous Region; The People's Hospital of Cangnan County, Zhejiang Province; The Second Hospital of Harbin City, Heilongjiang Province; The Fourth Affiliated Hospital of Harbin Medical University, Heilongjiang Province; Shanghai Fifth People's Hospital, Fudan University, Shanghai City; Fujian Provincial People's Hospital, Fujian Province; Liuzhou City Liutie Central Hospital, Guangxi Zhuang Autonomous Region; XinSteel Center Hospital, Jiangxi Province; Liaocheng Hospital of Traditional Chinese Medicine, Shandong Province; Cangzhou People's Hospital, Hebei Province; The First Hospital of Lanzhou University, Gansu Province; Xinzhou People's Hospital, Shanxi Province; Affiliated Hospital of Guangdong Medical University, Guangdong Province; The Sixth Hospital of Beijing, Beijing City; Panjin Liaoyou Gem Flower Hospital (Liaohe Oilfield General Hospital), Liaoning Province; The People's Hospital of Nanping City, Fujian Province; The First People's Hospital of Qinzhou, Guangxi Zhuang Autonomous Region; The First Hospital of Tianjin, Tianjin City; Yichun People's Hospital, Jiangxi Province; The Central Hospital of Ningcheng County, Inner Mongolia Autonomous Region; The First Affiliated Hospital of Bengbu Medical College, Anhui Province; The Second People's Hospital of Lianyungang, Jiangsu Province; Gansu Gem Flower Hospital, Gansu Province; The People's Hospital of Lhasa, Tibet Autonomous Region; Anhui No. 2 Provincial People's Hospital, Anhui Province; Qingdao Municipal Hospital, Shandong Province; Maoming Hospital of Traditional Chinese Medicine, Guangdong Province; The People's Hospital of Pingliang, Gansu Province; Guizhou Provincial People's Hospital, Guizhou Province; Zhangjiajie City People's Hospital, Hunan Province; The Affiliated Hospital of Inner Mongolia Medical University, Inner Mongolia Autonomous Region; First Hospital of Qinhuangdao, Hebei Province; Chifeng Municipal Hospital, Inner Mongolia Autonomous Region; Jiading District Central Hospital, Shanghai City; The First Affiliated Hospital of Anhui University of Traditional Chinese Medicine, Anhui Province; The Affiliated Hospital of Shandong University of Traditional Chinese Medicine (Shandong Provincial Hospital of Traditional Chinese Medicine), Shandong Province; Yanbian No. 2 People's Hospital, Jinlin Province; The Third People's Hospital of Yichang City, Hubei Province; The Second Affiliated Hospital 
of Liaoning University of Traditional Chinese Medicine, Liaoning Province; Shenzhen Traditional Chinese Medicine Hospital, Guangdong Province; The First People's Hospital of Jiangxia District, Wuhan City, Hubei Province; The First People's Hospital of Xining, Qinghai Province; Xiamen Haicang Hospital, Fujian Province; Tibet Autonomous Region People's Hospital, Tibet Autonomous Region; The Third Hospital of Xiamen, Fujian Province; Nanjing Jiangning District Hospital of Traditional Chinese Medicine, Jiangsu Province; Sichuan Academy of Medical Sciences, Sichuan Provincial People's Hospital (East Hospital), Sichuan Province; Wuzhou Gongren Hospital, Guangxi Zhuang Autonomous Region; Wang Jing Hospital of China Academy of Chinese Medical Sciences, Beijing City; Shanghai Seventh People's Hospital, Shanghai City; The Fifth People's Hospital of Chongqing City, Chongqing City; The People's Hospital of Dongying City, Shandong Province; The Affiliated Hospital of Hangzhou Normal University, Zhejiang Province; The First Affiliated Hospital of Shantou University Medical College, Guangdong Province; Xinyu Hospital of Traditional Chinese Medicine, Jiangxi Province; Xishan Coal Electricity Group Gujiao Mining Area General Hospital, Shanxi Province; Ruikang Hospital Affiliated to Guangxi Universtiy of Chinese Medicine, Guangxi Zhuang Autonomous Region; The Third Affiliated Hospital of Guangzhou Medical University, Guangdong Province; Yu Tian Xian Zhong Yi Yuan, Hebei Province; People's Hospital of Changshou Chongqing, Chongqing City; Beijing Tiantan Hospital, Capital Medical University, Beijing City; General Hospital of Heilongjiang Provincial Agricultural Reclamation Bureau, Heilongjiang Province; The People's Hospital of Xishui County, Hubei Province; The First Hospital of Hunan University of Chinese Medicine, Hunan Province; The First People's Hospital of Huainan City, Anhui Province; The Second Affiliated Hospital of Nanjing Medical University, Jiangsu Province; Shanxi Provincial People's Hospital, Shanxi Province; West China Hospital of Sichuan University, Sichuan Province; Zhongshan Hospital Xiamen University, Fujian Province; Zheng Zhou Shi Zhong Yi Yuan, Henan Province; Xiangya Ping Mine Cooperative Hospital, Jiangxi Province; Northen Jiangsu People's Hospital, Jiangsu Province; Huzhou Hospital of Traditional Chinese Medicine affiliated to Zhejiang University of Traditional Chinese Medicine, Zhejiang Province; Guangdong Second Provincial General Hospital (Guangdong Provincial Emergency Hospital), Guangdong Province; Huainan Xinhua Hospital, Anhui Province; Third People's Hospital of Jiujiang City, Jiangxi

\section{REFERENCES}

1. Poh TY, Mac Aogáin M, Chan AK, Yii AC, Yong VF, Tiew PY, et al. Understanding COPD-overlap syndromes. Expert Rev Respir Med. (2017) 11:285-98. doi: 10.1080/17476348.2017.13 05895

2. Bateman ED, Reddel HK, van Zyl-Smit RN, Agusti A. The asthmaCOPD overlap syndrome: towards a revised taxonomy of chronic airways diseases? Lancet Respir Med. (2015) 3:719-28. doi: 10.1016/ S2213-2600(15)00254-4

3. Postma DS, Rabe KF. The asthma-COPD overlap syndrome. N Engl J Med. (2015) 373:1241-9. doi: 10.1056/NEJMra1411863
Province; Panzhihua Hospital of Integrated Traditional Chinese and Western Medicine, Sichuan Province; The Central Hospital of Xuhui District, Shanghai City; Yueyang Integrated Traditional Chinese and Western Medicine Hospital Affiliated to Shanghai University of Traditional Chinese Medicine, Shanghai City; The Second Affiliated Hospital of Xiamen Medical College, Fujian Province; Hegang People's Hospital, Heilongjiang Province; Jiaozuo People's Hospital, Henan Province; Traditional Chinese Medicine Hospital of Kunshan, Jiangsu Province; The First People's Hospital of Nanning, Guangxi Zhuang Autonomous Region; Shandong Provincial Qianfoshan Hospital, Shandong Province; Beijing Hospital of Traditional Chinese Medicine, Capital Medical University, Beijing City; Tangshan People's Hospital, Hebei Province; Chongqing Traditional Chinese Medicine Hospital, Chongqing City.

\section{AUTHOR CONTRIBUTIONS}

JL conceptualized this study, did all statistical analyses, interpreted the data, wrote the first draft, and critically revised the manuscript. $\mathrm{CL}, \mathrm{KH}$, and $\mathrm{SW}$ took part in manuscript revision, project, and data management. TY and CW supervised the work, had full access to all of the data in the study, and took responsibility for the integrity of the work as a whole, from inception to the published article. All authors have read and approved the final manuscript to be published.

\section{FUNDING}

This work was supported by the Chinese Academy of Medical Science (CAMS) Innovation Fund for Medical Sciences (CIFMS) (nos. 2021-I2M-1-049 and 2020-I2M-2-008) and the Chinese Academy of Medical Science (CAMS) Basic Scientific Research Business Fee Fund of Central Level Public Welfare Scientific Research Institutes (no. 2019TX320005). The funders had no role in study design, data collection and analysis, decision to publish, or preparation of the manuscript.

\section{SUPPLEMENTARY MATERIAL}

The Supplementary Material for this article can be found online at: https:/www.frontiersin.org/articles/10.3389/fmed. 2022.817048/full\#supplementary-material
4. Sin DD, Miravitlles M, Mannino DM, Soriano JB, Price D, Celli BR, et al. What is asthma-COPD overlap syndrome? Towards a consensus definition from a round table discussion. Eur Respir J. (2016) 48:66473. doi: 10.1183/13993003.00436-2016

5. Leung JM, Sin DD. Asthma-COPD overlap syndrome: pathogenesis, clinical features, and therapeutic targets. BMJ. (2017) 358:j3772. doi: 10.1136/bmj.j3772

6. Bacharier LB, Mori A, Kita H. Advances in asthma, asthma-COPD overlap, and related biologics in 2018. J Allergy Clin Immunol. (2019) 144:90619. doi: 10.1016/j.jaci.2019.08.021

7. Marcon A, Locatelli F, Dharmage SC, Svanes C, Heinrich J, Leynaert $\mathrm{B}$, et al. The coexistence of asthma and COPD: risk factors, clinical 
history and lung function trajectories. Eur Respir J. (2021) 58:2004656. doi: 10.1183/13993003.04656-2020

8. Reddel HK, Vestbo J, Agustí A, Anderson GP, Bansal AT, Beasley $\mathrm{R}$, et al. Heterogeneity within and between physician-diagnosed asthma and/or COPD: NOVELTY cohort. Eur Respir J. (2021) 58:2003927. doi: 10.1183/13993003.03927-2020

9. Global Initiative for Asthma. Global Strategy for Asthma Management and Prevention, 2021. (2021). Available online at: www.ginasthma.org (accessed June 1, 2021).

10. Global Initiative for Chronic Obstructive Lung Disease. Global Strategy for the Diagnosis, Management, and Prevention of Chronic Obstructive Pulmonary Disease, 2021. (2021). Available online at: www.goldcopd.org (accessed January 1, 2021).

11. Leung C, Sin DD. Asthma-COPD Overlap: what are the important questions? Chest. (2022) 161:330-44. doi: 10.1016/j.chest.2021.09.036

12. O'Donnell AE. Bronchiectasis in patients with COPD: a distinct COPD phenotype? Chest. (2011) 140:1107-8. doi: 10.1378/chest.11-1484

13. Stockley RA. Bronchiectasis with chronic obstructive pulmonary disease: association or a further phenotype? Am J Respir Crit Care Med. (2013) 187:786-8. doi: 10.1164/rccm.201302-0203ED

14. Blasi F, Chalmers JD, Aliberti S. COPD and bronchiectasis: phenotype, endotype or co-morbidity? COPD. (2014) 11:6034. doi: 10.3109/15412555.2014.974744

15. Hurst JR, Elborn JS, De Soyza A. COPD-bronchiectasis overlap syndrome. Eur Respir J. (2015) 45:310-3. doi: 10.1183/09031936.00170014

16. Stockley RA. Bronchiectasis: a progressive phenotype of chronic obstructive pulmonary disease. Clin Infect Dis. (2021) 72:411-3. doi: 10.1093/cid/ciaa073

17. Alshabanat A, Zafari Z, Albanyan O, Dairi M, FitzGerald JM. Asthma and COPD overlap syndrome (ACOS): a systematic review and meta analysis. PLoS ONE. (2015) 10:e0136065. doi: 10.1371/ journal.pone. 0136065

18. Hosseini M, Almasi-Hashiani A, Sepidarkish M, Maroufizadeh S. Global prevalence of asthma-COPD overlap (ACO) in the general population: a systematic review and meta-analysis. Respir Res. (2019) 20:229. doi: 10.1186/s12931-019-1198-4

19. Zhou Y, Wang C, Yao W, Chen P, Kang J, Huang S, et al. COPD in Chinese nonsmokers. Eur Respir J. (2009) 33:509-18. doi: 10.1183/09031936.00084408

20. Ding B, DiBonaventura M, Karlsson N, Ling X. Asthma-chronic obstructive pulmonary disease overlap syndrome in the urban Chinese population: prevalence and disease burden using the 2010, 2012 and 2013. China National Health and Wellness Surveys. Int J Chronic Obstruct Pulmonary Dis. (2016) 11:1139-50. doi: 10.2147/COPD.S103873

21. Uchida A, Sakaue K, Inoue H. Epidemiology of asthma-chronic obstructive pulmonary disease overlap (ACO). Allergol Int. (2018) 67:165-71. doi: 10.1016/j.alit.2018.02.002

22. Ni Y, Shi G, Yu Y, Hao J, Chen T, Song H. Clinical characteristics of patients with chronic obstructive pulmonary disease with comorbid bronchiectasis: a systemic review and meta-analysis. Int J Chron Obstruct Pulmon Dis. (2015) 10:1465-75. doi: 10.2147/COPD.S83910

23. Athanazio R. Airway disease: similarities and differences between asthma, COPD and bronchiectasis. Clinics. (2012) 67:133543. doi: 10.6061/clinics/2012(11)19

24. Polverino E, Dimakou K, Hurst J, Martinez-Garcia MA, Miravitlles M, Paggiaro $\mathrm{P}$, et al. The overlap between bronchiectasis and chronic airway diseases: state of the art and future directions. Eur Respir J. (2018) 52:1800328. doi: $10.1183 / 13993003.00328-2018$

25. Kumbhare S, Pleasants R, Ohar JA, Strange C. Characteristics and prevalence of asthma/chronic obstructive pulmonary disease overlap in the United States. Ann Am Thorac Soc. (2016) 13:803-10. doi: 10.1513/AnnalsATS.201508-554OC

26. Vaz Fragoso CA, Murphy TE, Agogo GO, Allore HG, McAvay GJ. AsthmaCOPD overlap syndrome in the US: a prospective population-based analysis of patient-reported outcomes and health care utilization. Int J Chron Obstruct Pulmon Dis. (2017) 12:517-27. doi: 10.2147/COPD.S121223

27. Park HJ, Byun MK, Kim HJ, Ahn CM, Lee JH, Shin KC, et al. AsthmaCOPD overlap shows favorable clinical outcomes compared to pure COPD in a Korean COPD cohort. Allergy Asthma Immunol Res. (2017) 9:4317. doi: 10.4168/aair.2017.9.5.431
28. Yamauchi Y, Yasunaga H, Matsui H, Hasegawa W, Jo T, Takami K, et al. Comparison of in-hospital mortality in patients with COPD, asthma and asthma-COPD overlap exacerbations. Respirology. (2015) 20:9406. doi: 10.1111/resp.12556

29. Bai JW, Mao B, Yang WL, Liang S, Lu HW, Xu JF. Asthma-COPD overlap syndrome showed more exacerbations however lower mortality than COPD. QJM. (2017) 110:431-6. doi: 10.1093/qjmed/hcx005

30. Du Q, Jin J, Liu X, Sun Y. Bronchiectasis as a comorbidity of chronic obstructive pulmonary disease: a systematic review and meta-analysis. PLoS ONE. (2016) 11:e0150532. doi: 10.1371/journal.pone.0150532

31. Pei Z, Sun Y, Wang S, Chen Y, Yang T, Huang K, et al. Estimating mortality among inpatients with acute exacerbation of chronic obstructive pulmonary disease using registry data. NPJ Primary Care Respir Med. (2020) 30:28. doi: 10.1038/s41533-020-0186-y

32. Liang C, Mao X, Niu H, Huang K, Dong F, Chen Y, et al. Characteristics, management and in-hospital clinical outcomes among inpatients with acute exacerbation of chronic obstructive pulmonary disease in china: results from the phase i data of ACURE study. Int J Chron Obstruct Pulmon Dis. (2021) 16:451-65. doi: 10.2147/COPD.S281957

33. Polverino E, Goeminne PC, McDonnell MJ, Aliberti S, Marshall $\mathrm{SE}$, Loebinger MR, et al. European respiratory society guidelines for the management of adult bronchiectasis. Eur Respir J. (2017) 50:1700629. doi: 10.1183/13993003.00629-2017

34. Pasteur MC, Bilton D, Hill AT. British thoracic society guideline for non-CF bronchiectasis. Thorax. (2010) 65(Suppl. 1):i1-58. doi: 10.1136/thx.2010.136119

35. Bellou V, Belbasis L, Konstantinidis AK, Tzoulaki I, Evangelou E. Prognostic models for outcome prediction in patients with chronic obstructive pulmonary disease: systematic review and critical appraisal. BMJ. (2019) 367:15358. doi: 10.1136/bmj.15358

36. Song P, Zha M, Xia W, Zeng C, Zhu Y. Asthma-chronic obstructive pulmonary disease overlap in China: prevalence, associated factors and comorbidities in middle-aged and older adults. Curr Med Res Opin. (2020) 36:66775. doi: 10.1080/03007995.2020.1722082

37. Cosio BG, Soriano JB, López-Campos JL, Calle-Rubio M, Soler-Cataluna JJ, de-Torres JP, et al. Defining the asthma-COPD overlap syndrome in a COPD cohort. Chest. (2016) 149:45-52. doi: 10.1378/chest.15-1055

38. Hansen JE, Dilektasli AG, Porszasz J, Stringer WW, Pak Y, Rossiter $\mathrm{HB}$, et al. A new bronchodilator response grading strategy identifies distinct patient populations. Ann Am Thorac Soc. (2019) 16:150417. doi: 10.1513/AnnalsATS.201901-030OC

39. Fortis S, Comellas A, Make BJ, Hersh CP, Bodduluri S, Georgopoulos D, et al. Combined forced expiratory volume in 1 second and forced vital capacity bronchodilator response, exacerbations, and mortality in chronic obstructive pulmonary disease. Ann Am Thorac Soc. (2019) 16:826-35. doi: 10.1513/AnnalsATS.201809-601OC

40. Chhabra SK. Acute bronchodilator response has limited value in differentiating bronchial asthma from COPD. J Asthma. (2005) 42:367-72. doi: 10.1081/JAS-200062992

Conflict of Interest: The authors declare that the research was conducted in the absence of any commercial or financial relationships that could be construed as a potential conflict of interest.

Publisher's Note: All claims expressed in this article are solely those of the authors and do not necessarily represent those of their affiliated organizations, or those of the publisher, the editors and the reviewers. Any product that may be evaluated in this article, or claim that may be made by its manufacturer, is not guaranteed or endorsed by the publisher.

Copyright $\odot 2022$ Lei, Yang, Liang, Huang, Wu and Wang. This is an open-access article distributed under the terms of the Creative Commons Attribution License (CC $B Y)$. The use, distribution or reproduction in other forums is permitted, provided the original author(s) and the copyright owner(s) are credited and that the original publication in this journal is cited, in accordance with accepted academic practice. No use, distribution or reproduction is permitted which does not comply with these terms. 